\title{
Analysis of Reporting Adverse Drug Reactions in Paediatric Patients in a University Hospital in the Netherlands
}

\author{
Anne T. M. Dittrich ${ }^{1,2}$ (D) Jos M. T. Draaisma ${ }^{1}$ Eugène P. van Puijenbroek ${ }^{3,4} \cdot$ D. Maroeska W. M. te Loo ${ }^{2,5}$
}

Published online: 18 June 2020

(c) The Author(s) 2020

\begin{abstract}
Aims The risk to develop adverse drug reactions (ADRs) is high for paediatric patients. This is, amongst other reasons, due to the inevitable use of off-label and unlicensed medicines. Moreover, there is limited knowledge on ADRs in children. Thus, adequate recognition may be challenging. The lack of dedicated studies and the voluntary nature of pharmacovigilance systems used to gain insight into the characteristics of ADRs contribute to this problem. The goal of this study is to identify whether ADRs in paediatric patients are adequately documented by the medical team and whether they are subsequently reported to the national pharmacovigilance system.

Methods All patients admitted to the paediatric medium care of the Radboudumc Amalia Children's hospital during 1 month, and using one or more drugs, were included. Two researchers analysed retrospectively and independently the number of possible ADRs in the medical records. The ADRs were listed per paediatric subspecialty, to evaluate any differences in documentation and reporting of the ADRs. Subsequently, the causality, severity, and seriousness of the ADRs were assessed. The ADRs were categorised by system organ class and drug class. The national pharmacovigilance centre was consulted to check if there were any reports coming from our hospital and to collect the total number of reports.

Results The medical records of 301 patients were analysed, 81 patients were suffering from one or more ADRs. In total 132 suspected ADRs were found, divided among 19 different paediatric subspecialties. Numbers were too small to investigate the differences in ADR documentation. Of these found ADRs, 55\% were not explicitly noted as such in the medical records by the treating physician. None of the ADRs were reported to the national pharmacovigilance centre. Most ADRs scored 'possible' in the causality assessment, were mild or moderate, and a small number were serious. The ADRs occurred in 25 different organ systems. In total 25 different drug classes were involved.

Conclusions The results of the present study show that a large number of ADRs are not registered in the medical records and are not reported to the national pharmacovigilance system. Furthermore, it is shown that the number of ADRs occurring at our centre is much higher than the number reported to the national pharmacovigilance centre. Only an average of 513 ADRs in paediatric patients are reported per year nationwide, suggesting that there is extensive underreporting.
\end{abstract}

\section{Introduction}

Drug safety is an essential aspect of health care, and knowledge of adverse drug reactions (ADRs) is important for preventing unnecessary harm [1]. This is especially true in the paediatric population where medication is often used off-label or unlicensed [2, 3]. However, data on ADRs in children are limited for a number of reasons. Premarketing clinical trials are predominantly performed in adults,

Anne T. M. Dittrich

anne.dittrich@radboudumc.nl

Extended author information available on the last page of the article and data regarding the safety of drugs that are obtained in adults cannot be extrapolated to paediatric patients because both the pharmacokinetics and pharmacodynamics differ substantially between the two populations [4]. If children are included in premarketing studies, the studies generally involve only small groups of patients, making it difficult to interpret the true extent of ADRs. Furthermore, many drugs prescribed to children on a regular basis are drugs that have been on the market for a long period of time. Since a peak in ADR reporting for drugs that were recently marketed (the Weber effect) may exist [5], for drugs that have been on the market for a long time, there is less emphasis on postmarketing surveillance. However, there is a clear need to improve the recognition, registration and reporting of ADRs 


\section{Key Points}

Twenty-six per cent of paediatric patients admitted to the medium care ward developed adverse drug reactions (ADRs). Fifty-five per cent of possible ADRs were not documented in the medical records, and none of the ADRs were reported to the national pharmacovigilance centre.

Underreporting of ADRs in paediatric patients in the Netherlands seems to be extensive.

To improve our knowledge of drugs used in paediatric patients, a system that assures adequate reporting of ADRs is needed.

in paediatric patients so as to increase the knowledge on the safety of drugs in this vulnerable group of patients.

The lack of knowledge on ADRs in paediatric pharmacotherapy is partly caused by off-label and unlicensed drug use. In paediatrics, this is unavoidable because there is often no on-label alternative. Estimations of unlicensed and offlabel prescriptions vary greatly and depend on the type of ward and definitions used [2, 6-10]. Several studies have shown that the risk of ADRs is higher for unlicensed or offlabel prescriptions $[2,9,10]$. Cuzzolin et al. reviewed nine studies to investigate the safety of off-label and unlicensed drug use in paediatrics [2]. Among these nine studies, the percentage of unlicensed and/or off-label prescriptions involved in an ADR ranged between 23 and 60\% [2].

The development and testing of paediatric medicines in Europe improved over the last decade. A major contribution to this development came from 'The Paediatric Regulation' [11] by the European Union. Despite improved paediatric medicine legislation, the systematic registration of ADRs in the postmarketing phase is still wanting, especially for rare and severe drug reactions. It is therefore of the utmost importance that all severe and rare ADRs occurring in paediatric patients are reported to the national pharmacovigilance system. To achieve this goal, ADRs must be recognised adequately by health care professionals and reported to the responsible authorities or the responsible marketing authorisation holders. Like in many other countries, in the Netherlands, a nationwide, voluntary pharmacovigilance system is in place. Its primary goal is to signal hitherto unknown risks associated with the use of medicines in daily practice. In most cases, additional studies are needed to confirm these safety signals. Physicians, pharmacologists, nurses, patients, and their parents/caregivers can report ADRs on a voluntary basis. Underreporting is a widely recognised problem in these voluntary reporting schemes [12] and leads to a possible lack of knowledge with regard to adverse reactions to prescribed drugs in paediatric patients. The extent to which underreporting occurs, however, is not known.

We performed a retrospective, cross-sectional study at our university hospital. The primary aims were to identify whether ADRs in paediatric patients were adequately documented by the medical team and whether these ADRs were subsequently reported to the national pharmacovigilance centre in the Netherlands. The secondary aims were to assess the causality and severity of the found ADRs, the classes of drugs that caused the reactions, and the possible differences in ADR documenting between the paediatric subspecialties.

\section{Methods}

We analysed retrospectively the ADRs occurring in the paediatric medium care wards of the Amalia Children's Hospital at the Radboud University Medical Centre during a randomly chosen period of 1 month. This month was June 2016. Patients from 0 to 18 years old who were using one or more drugs were included. Patients admitted to the high care and the intensive care units were not included because of the distinct characteristics of those populations concerning the level of illness and the types of drugs used. All medical records and hospital charts of the included patients were analysed retrospectively and manually by two independent clinical pharmacologists. They looked for ADRs and whether the ADRs were mentioned in the medical records by the treating physicians. Specifically, the researchers looked for signs, symptoms, and deviating laboratory or radiology results which, in combination with the drugs the patients were using, could be considered an ADR as defined by the World Health Organization (WHO), European Union, and the European Medicine Agency (EMA). They define an ADR as 'a response to a medicinal product which is noxious and unintended. Adverse reactions may arise from use of the product within or outside the terms of the marketing authorisation or from occupational exposure. Use outside the marketing authorisation includes off-label use, overdose, misuse, abuse and medication errors' [13-15]. No distinction was made regarding duration of use of the drug or duration of the ADR. In other words, all ADRs were taken into account: ADRs which occurred before hospitalisation, but continued during hospitalisation (not necessarily being the reason for the admission) and ADRs that occurred during hospitalisation.

In case there was a difference in assessment between the two independent researchers, a third qualified researcher was asked to study the patients' records. ADRs were assessed during the complete admission period in the medium care ward, even if this period extended beyond the chosen month.

Subsequently, the causality of the found ADRs was assessed according to the WHO-Uppsala Monitoring Centre 
(UMC) system [16], and the severity of the found ADRs was assessed according to the Common Terminology Criteria for Adverse Events (CTCAE) scale [17]. More information about the CTCAE scale is shown in Table 1. Seriousness was assessed according to the EMA International Conference on Harmonization (ICH) E2A guideline [18, 19]. More information about the criteria from this guideline concerning the seriousness of an ADR is shown in Table 2. Furthermore, the ADRs were categorised by System Organ Class (System Organ Class is identified by anatomical or physiological system, aetiology, or purpose) [17] and the medicines responsible for the ADRs were categorised. Also, the number of ADRs was listed per paediatric subspecialty to which the child was admitted at the Amalia Children's Hospital and the number of reported ADRs was compared to the number of ADR reports that were submitted to the national pharmacovigilance centre in the Netherlands. There were no ADRs caused by vaccines in this study since vaccines are generally not administered in our hospital. In this study period, no vaccines were given.

\section{Results}

In 2016, a total of 4032 paediatric patients were admitted to the medium care wards in our hospital (average per month 336). Divided among 19 different paediatric subspecialties, a total of 315 children were hospitalised in June 2016. The median age of the patients was 5 years (interquartile range 1-11 years). The median length of a patient's stay was 3 days (interquartile range 2-6 days). Of the 315 patients, $301(96 \%)$ received one or more drugs and were included in this study.

In the medical records of 81 patients (26\%), we found evidence of ADRs. In total, 132 suspected ADRs were found. Some of the patients suffered from more than one ADR at the same time; eight patients had two ADRs, three patients had three ADRs, three patients had four ADRs, one patient had seven ADRs, and two patients had eight ADRs. The patients suffering from multiple ADRs all had cancer and received chemotherapy.

Table 1 Grade refers to the severity of the adverse drug reaction (ADR). The CTCAE displays grades 1 through 5 with unique clinical descriptions of severity for each ADR based on this general guideline [17]

\begin{tabular}{ll}
\hline Grade & Description \\
\hline Grade 1 & Mild; asymptomatic or mild symptoms; clinical or diagnostic observations only; intervention not indicated \\
Grade 2 & Moderate; minimal, local or noninvasive intervention indicated; limiting age-appropriate instrumental ADL \\
Grade 3 & Severe or medically significant, but not immediately life-threatening; hospitalisation or prolongation of \\
& hospitalisation indicated; disabling; limiting self care ADL \\
Grade 4 & Life-threatening consequences; urgent intervention indicated \\
Grade 5 & Death related to ADR \\
\hline
\end{tabular}

$A D L$ activities of daily living, CTCAE Common Terminology Criteria for Adverse Events

${ }^{a}$ Instrumental ADL refer to preparing meals, shopping for groceries or clothes, using the telephone, managing money, etc.

${ }^{\mathrm{b}}$ Self care ADL refer to bathing, dressing and undressing, feeding self, using the toilet, taking medications, and not bedridden

Table 2 Criteria of a serious adverse drug reaction, according to the ICH E2A guideline [18, 19]

In accordance with the ICH E2A guideline, a serious adverse event or reaction is any untoward medical occurrence that at any dose:

Results in death

Is life-threatening ${ }^{\mathrm{a}}$

Requires inpatient hospitalisation or results in prolongation of existing hospitalisation

Results in persistent or significant disability/incapacity

Is a congenital anomaly/birth defect

Is a medically important event or reaction ${ }^{\mathrm{b}}$

ICH International Conference on Harmonization

aThe term 'life-threatening' in the definition of 'serious' refers to an event/reaction in which the patient was at risk of death at the time of the event/reaction; it does not refer to an event/reaction which hypothetically might have caused death if it were more severe)

${ }^{b}$ Medical and scientific judgment should be exercised in deciding whether other situations should be considered serious such as important medical events that might not be immediately life-threatening or result in death or hospitalisation but might jeopardise the patient or might require intervention to prevent one of the other outcomes listed in the definition above. Examples of such events are intensive treatment in an emergency room or at home for allergic bronchospasm, blood dyscrasias or convulsions that do not result in hospitalisation, or development of drug dependency or drug abuse 
Of those suspected ADRs, 73 (55\%) were not noted as such in the medical records by the physicians who cared for the patients at the time. Ninety per cent of the ADRs reported by the two independent researchers were in line with each other. In $10 \%$ of the cases, the opinion of the third researcher was needed.

According to the WHO-UMC system [16], 30 ADRs were considered 'certain', 29 'probable', 61 'possible', and three 'unlikely'. Nine were considered 'not assessable', due to missing information. The ADRs were categorised by group of paediatric disciplines. The number of hospitalised patients, the number of suspected ADRs, and the proportion of ADRs recorded in the medical records varied considerably. For example, in the oncology group, 56 ADRs were found in 31 patients. In other disciplines, this number was far less. For example, in the 'small surgery' group, only four suspected ADRs were found in 35 patients. This group had the lowest ADR reporting rate. Only one of the four ADRs was noted explicitly in the medical records by the treating physician. In the urinary tract group, this number was seven out of eight (88\%) [Table 3].

The ADRs were divided by 15 different systems according to the System Organ Class (Table 4). Twenty-five different classes of drugs were responsible for the 132 possible ADRs. They are shown in Table 5. In order of associated number of ADRs, cytostatic drugs, followed by antibiotics then anaesthetics, ranked highest.

According to the CTCAE criteria [17], 62 ADRs were grade 1, 54 ADRs were grade 2, 14 ADRs were grade 3, and two ADRs were grade 4 (Table 4). None of the found ADRs were more severe than grade 4 . The two grade 4 ADRs were 'low white cell count' caused by chemotherapy. Ten of the grade 3 ADRs were febrile neutropenia or low white cell count, all caused by chemotherapy. The other four grade 3 ADRs were an allergic reaction to platelet transfusion for which medication was necessary, encephalopathy due to overdosing with acyclovir, prolonged hospitalisation due to severe nausea after anaesthesia, and severe oral mucositis after chemotherapy.

According to the ICH E2A guideline [18, 19], 16 ADRs were serious (Table 4). All grade 3 and grade 4 ADRs were also serious ADRs, with two exceptions; two patients with low blood count due to chemotherapy scored grade 3 at the severity scale, but this did not affect their discharge from the hospital and were therefore not scored as 'serious'. In two other cases, the opposite happened; one patient suffered from extrapyramidal disorders caused by haloperidol and one patient suffered from fever after the use of interferon. Both ADRs scored grade 2 for severity, but for both patients, the occurrence of the ADRs resulted in prolongation of their hospitalisation.

The number of ADRs reported to the national pharmacovigilance centre over a period of 3 years, specifically from January 1, 2015 until December 31, 2017, was analysed. None of the ADRs identified in the present study were reported to the national pharmacovigilance centre. In 2015, 2016, and 2017, the national pharmacovigilance centre received 511, 504, and 526 reports on ADRs in children that were treated in the Netherlands, respectively. In June 2016, the national pharmacovigilance centre received only one report of a suspected ADR in a paediatric patient from our university hospital. This patient, however, was not hospitalised in June, but visited the outpatient clinic and was therefore not a subject in our study. Notably, the most

Table 3 Overview of the patients hospitalised and the number of adverse drug reactions (ADRs), distributed by hospital wards

\begin{tabular}{|c|c|c|c|c|c|c|c|c|}
\hline & \multicolumn{2}{|c|}{$\begin{array}{l}\text { Number of hospitalised } \\
\text { patients using medication }\end{array}$} & \multicolumn{2}{|c|}{ Total number of ADRs } & \multicolumn{2}{|c|}{$\begin{array}{l}\text { ADRs documented by treat- } \\
\text { ing physician }\end{array}$} & \multicolumn{2}{|c|}{$\begin{array}{l}\text { ADRs not documented } \\
\text { by treating physician }\end{array}$} \\
\hline & Number & $\%$ & Number & $\%$ & Number & $\%$ & Number & $\%$ \\
\hline General surgery ${ }^{\mathrm{a}}$ & 74 & $24.6 \%$ & 14 & $10.6 \%$ & 4 & $28.6 \%$ & 10 & $71.4 \%$ \\
\hline General paediatrics ${ }^{\mathrm{b}}$ & 52 & $17.3 \%$ & 25 & $19.0 \%$ & 9 & $36.0 \%$ & 16 & $64.0 \%$ \\
\hline Urinary $\operatorname{tract}^{\mathrm{c}}$ & 47 & $15.6 \%$ & 8 & $6.1 \%$ & 7 & $87.5 \%$ & 1 & $12.5 \%$ \\
\hline Nervous system ${ }^{\mathrm{d}}$ & 42 & $14.0 \%$ & 19 & $14.4 \%$ & 13 & $68.4 \%$ & 6 & $31.6 \%$ \\
\hline Small surgery ${ }^{\mathrm{e}}$ & 36 & $12.0 \%$ & 4 & $3.0 \%$ & 1 & $25.0 \%$ & 3 & $75.0 \%$ \\
\hline Oncology $\mathrm{y}^{\mathrm{f}}$ & 31 & $10.3 \%$ & 56 & $42.4 \%$ & 22 & $39.3 \%$ & 34 & $60.7 \%$ \\
\hline Cardiology & 19 & $6.3 \%$ & 6 & $4.5 \%$ & 3 & $50.0 \%$ & 3 & $50.0 \%$ \\
\hline Total & 301 & & 132 & & 59 & $44.7 \%$ & 73 & $55.3 \%$ \\
\hline
\end{tabular}

${ }^{a}$ Includes orthopaedic surgery

${ }^{\mathrm{b}}$ Includes endocrinology, gastro-enterology, metabolic disease and pulmonology

${ }^{\mathrm{c}}$ Includes nephrology and urology

${ }^{\mathrm{d}}$ Includes neurology and neurosurgery

${ }^{\mathrm{e}}$ Includes otorhinolaryngology, ophthalmology, maxillofacial surgery, plastic surgery, and radiology (intervention)

f Includes haematology 
Table 4 Overview of the severity (CTCAE scale) and seriousness of the found adverse drug reactions (ADRs), divided by system organ class

\begin{tabular}{|c|c|c|c|c|c|c|}
\hline System organ class & $\begin{array}{l}\text { Total number } \\
\text { ADRs }\end{array}$ & $\begin{array}{l}\text { CTCAE grade } \\
1\end{array}$ & $\begin{array}{l}\text { CTCAE grade } \\
2\end{array}$ & $\begin{array}{l}\text { CTCAE grade } \\
3\end{array}$ & $\begin{array}{l}\text { CTCAE grade } \\
4\end{array}$ & Serious ADRs \\
\hline Blood and lymphatic disorders & 8 & - & - & 8 & - & 8 \\
\hline Cardiac disorders & 3 & 3 & - & - & - & - \\
\hline Gastrointestinal disorders & 44 & 26 & 16 & 2 & - & 2 \\
\hline General disorders & 6 & 2 & 4 & - & - & 1 \\
\hline Immune system & 2 & - & 1 & 1 & - & 1 \\
\hline Investigations & 32 & 11 & 17 & 2 & 2 & 2 \\
\hline Metabolism and nutrition & 2 & - & 2 & - & - & - \\
\hline Muskoskeletal disorders & 2 & 1 & 1 & - & - & - \\
\hline Nervous system disorders & 12 & 5 & 6 & 1 & - & 2 \\
\hline Psychiatric disorders & 5 & 2 & 3 & - & - & - \\
\hline Renal and urinary disorders & 1 & 1 & - & - & - & - \\
\hline Respiratory disorders & 1 & 1 & - & - & - & - \\
\hline Skin disorders & 4 & 2 & 2 & - & - & - \\
\hline Vascular disorders & 10 & 8 & 2 & - & - & - \\
\hline Total & 132 & 62 & 54 & 14 & 2 & 16 \\
\hline
\end{tabular}

CTCAE Common Terminology Criteria for Adverse Events

Table 5 Drug classes responsible for adverse drug reactions (ADRs) in the present study

\begin{tabular}{lcl}
\hline Drug classes & Number of ADRs & $\begin{array}{l}\text { Proportion } \\
\text { of total } \\
(\%)\end{array}$ \\
\hline Cytostatics & 38 & 28.8 \\
Antibiotics & 12 & 9.1 \\
Anaesthetics & 11 & 8.3 \\
Steroids & 10 & 7.6 \\
Opioids & 10 & 7.6 \\
Anti-epileptics & 10 & 7.6 \\
Antimycotics & 8 & 6.1 \\
Other (18 classes) & 33 & 25.0 \\
Total & 132 & 100 \\
\hline
\end{tabular}

notifications sent to the national pharmacovigilance centre in 2016 were reported by patients or parents of patients $(n=215)$, followed by physicians $(n=199)$, pharmacists $(n=66)$, and nurses $(n=24)$.

\section{Discussion}

The risk to develop ADRs for paediatric patients is high, partly due to the high number of off-label and unlicensed drugs used in paediatrics. In our study, in $26 \%$ of the patients admitted in the study period, one or more possible ADRs were present. In total, we found 132 suspected ADRs. This result is similar when compared with results in previous literature [20, 21]. More than 50\% of the suspected ADRs identified by the researchers were not documented as such by the treating physician in the medical records.

Table 3 shows the difference in number of hospitalised patients and number of suspected ADRs between the paediatric disciplines. A secondary aim of the study was to investigate the differences in ADR documentation between the paediatric subspecialties. Unfortunately, the numbers are too small to draw conclusions and answer this question. However, we can conclude that most of the ADRs were found in oncology patients. Most of the patients with multiple ADRs were also oncology patients, and most ADRs were caused by cytostatics (Table 5 ).

None of the suspected ADRs we found in the study were reported to the Dutch pharmacovigilance centre, including the severe and serious ADRs. So questions arise as to whether the ADRs were not recognised, whether they were recognised but judged as not important enough to mention explicitly in the medical record, and whether only more severe ADRs would have been reported. Either way, this result indicates that there may be a lack of awareness and/or knowledge regarding the importance of reporting ADRs to the responsible authorities.

In the Netherlands, there are approximately 75 hospitals, most of which have paediatric departments. Our hospital is one of seven university hospitals in the country. The population of the hospital consists of patients from our region who need 'low complex care', and patients from all over the country who need 'high complex care'. In our hospital, approximately 4000-4500 paediatric patients are admitted every year to the medium care wards. In the Netherlands, 
every year approximately 720,000 paediatric patients are admitted to a hospital [22]. On average, only 513 ADRs per year are reported to the national pharmacovigilance centre in the Netherlands. In this study, we found 132 ADRs in 315 paediatric patients. These numbers indicate that ADRs are considerably underreported in our country despite the European Union regulation [11].

In daily practice, it is impossible to report all ADRs, so it may be a feasible alternative to focus on new, rare, and severe drug reactions, also as a countermeasure against the substantial underreporting of ADRs in paediatric patients suggested by our study.

In this study, the medical records were retrospectively studied. An advantage of this retrospective approach is that it allows for usual care. The treating physicians did not know about this study, so there was no information bias. The researchers looked at trigger tools from previous research $[23,24]$ before screening the medical reports in our study. We decided not to use a trigger tool, because we were afraid we would miss possible ADRs. We did look at all aspects mentioned in the trigger tools, namely used drugs, deviating laboratory results, and signs/symptoms written down by the medical team in patient records. A point of attention is that due to the limited amount of information noted in the medical records, assessment of the causal relationship between suspected drugs and clinical symptoms is difficult.

It is essential that the safety of all medicines is monitored as determined by European Union law [25]. The overall pharmacovigilance system could operate through the cooperation of patients, health care workers, national regulatory authorities, the European Member States, European Medicines Agency, and the European Commission. Nonetheless, all systems function less optimally if there is extensive underreporting.

Reasons for underreporting include a lack of information, awareness, and training programs for health care professionals; physicians experiencing difficulties in correctly diagnosing ADRs; physicians having little time for making reports, which is often considered an additional workload; the absence of formal pharmacovigilance systems; complicated reporting systems; problems connected with potential legal conflicts; and an absence of feedback systems [3, 26, 27]. Furthermore, if the ADR does not lead to prolonged hospitalisation or even worse, most physicians may not feel the importance of reporting as the patient suffered, in their opinion, no real disadvantageous effect. In particular, those that have had longer clinical practice recognise some ADRs as common even though they are not mentioned in pharmacological guidelines.

In this study, we showed 'we are not doing the right thing' when it comes to reporting ADRs. The main question is how can we make things better? For that we must increase medical personnel's awareness of ADRs and improve the pharmacovigilance system to better monitor the safety of drug use in paediatric patients. One of the possible solutions is the use of a more active surveillance system, similar to those, for example, in Canada, the United States, and China [28-31]. Voluntary reporting of ADRs is focused on generating potential signals (finding new information on side effects), but this system is dependent on the reports. When one can increase the number of ADR reports in a more active surveillance system, more information is gathered, so the knowledge on ADRs is increased faster. The first step to achieve this is the education of paediatric health care workers. Proof that further education leads to higher reporting rates comes from the example of Cuba. According to the review of Clif-Eribo et al. [32], the high reporting rates recorded in a regional ADR database in Cuba in various studies were attributed to a special educational program on pharmacovigilance for health care professionals [33, 34]. Of course, the sustainability of such practices needs to be guaranteed by having health care professionals repeat such a program and keeping them enthusiastic. Education regarding the importance of registering the occurrence of ADRs may change the opinions of medical personnel. Training more paediatricians to become clinical pharmacologists can also be helpful. Clinical pharmacologists have more expertise in ADRs and recognising them. If there are clinical pharmacologists among paediatricians in the different hospitals, more focus will be on pharmacology, including ADR recognition, documenting, and reporting, for example, during morning reports. Furthermore, if the time needed to report ADRs to the national pharmacovigilance centres is the main problem, support to fill out the forms that are needed for notifying a pharmacovigilance centre by, for example, data managers could overcome this problem. Additionally, use could be made of a 'trigger tool' to efficiently detect ADRs in medical records $[23,24]$. Furthermore, in this digital age, electronic tools will be developed that can detect ADRs in a smart way.

\section{Conclusion and Recommendations}

Our study shows that the documenting and reporting of ADRs in paediatrics need to be improved in the Netherlands. To achieve this, the two most important items are to improve awareness among health care personnel (with education and training, including training more clinical pharmacologists) and to make reporting of ADRs as easy as possible, with the support of electronic solutions and extra personnel, for example, research nurses or data managers. This may lead to an increase in drug safety in this vulnerable population.

Acknowledgements The authors want to acknowledge the following persons for their contribution to this study: Prof. Dr. B. Carleton, Dr. Y. Kroeze, S.J.F. Hartman MD, Dr. C.W. Ockeloen, and N.J.L. Smeets MD. 
Author contributions DL designed the present study. AD extracted/ collected the data. Subsequently, the analyses were performed by AD and DL. All authors contributed to the interpretation of the data. AD and DL are responsible for the quality of the data presented in the manuscript. AD drafted the manuscript. DL, JD, and EP reviewed, commented, and approved the final version of the manuscript.

\section{Compliance with Ethical Standards}

Conflict of interest Authors AD, JD, EP, and DM declare that they have no conflict of interest.

Funding No sources of funding were used to support the writing of this article. Open access funding was provided through an agreement between SpringerNature and the Association of Dutch Universities and the Koninklijke Nederlandse Akademie van Wetenschappen.

Ethics approval This is a retrospective study for which formal consent is not needed.

Data Availability Statement The data that support the findings of this study are available from the corresponding author upon reasonable request.

Open Access This article is licensed under a Creative Commons Attribution-NonCommercial 4.0 International License, which permits any non-commercial use, sharing, adaptation, distribution and reproduction in any medium or format, as long as you give appropriate credit to the original author(s) and the source, provide a link to the Creative Commons licence, and indicate if changes were made. The images or other third party material in this article are included in the article's Creative Commons licence, unless indicated otherwise in a credit line to the material. If material is not included in the article's Creative Commons licence and your intended use is not permitted by statutory regulation or exceeds the permitted use, you will need to obtain permission directly from the copyright holder. To view a copy of this licence, visit http://creativecommons.org/licenses/by-nc/4.0/.

\section{References}

1. Leendertse AJ, Egberts AC, Stoker LJ, van den Bemt PM. Frequency of and risk factors for preventable medication-related hospital admissions in the Netherlands. Arch Intern Med. 2008;168(17):1890-6. https://doi.org/10.1001/archintern med.2008.3.

2. Cuzzolin L, Atzei A, Fanos V. Off-label and unlicensed prescribing for newborns and children in different settings: a review of the literature and a consideration about drug safety. Exp Opin Drug Saf. 2006;5(5):703-18. https://doi.org/10.1517/14740 338.5.5.703.

3. Fabiano V, Mameli C, Zuccotti GV. Adverse drug reactions in newborns, infants and toddlers: pediatric pharmacovigilance between present and future. Exp Opin Drug Saf. 2012;11(1):95105. https://doi.org/10.1517/14740338.2011.584531.

4. Kearns GL, Abdel-Rahman SM, Alander SW, Blowey DL, Leeder JS, Kauffman RE. Developmental pharmacology-drug disposition, action, and therapy in infants and children. New Engl J Med. 2003;349(12):1157-67. https://doi.org/10.1056/ NEJMra035092.

5. Hartnell NR, Wilson JP. Replication of the Weber effect using postmarketing adverse event reports voluntarily submitted to the
United States Food and Drug Administration. Pharmacotherapy. 2004;24(6):743-9. https://doi.org/10.1592/phco.24.8.743.36068.

6. Moulis F, Durrieu G, Lapeyre-Mestre M. Off-label and unlicensed drug use in children population. Therapie. 2018;73(2):135-49. https://doi.org/10.1016/j.therap.2018.02.002.

7. Dornelles AD, Calegari LH, de Souza L, Ebone P, Tonelli TS, Carvalho CG. The unlicensed and off-label prescription of medications in general paediatric ward: an observational study. Curr Pediatr Rev. 2019;15(1):62-6. https://doi.org/10.2174/15733 96314666181113101506.

8. Teigen A, Wang S, Truong BT, Bjerknes K. Off-label and unlicensed medicines to hospitalised children in Norway. J Pharm Pharmacol. 2017;69(4):432-8. https://doi.org/10.1111/jphp.12581.

9. Choonara I, Conroy S. Unlicensed and off-label drug use in children: implications for safety. Drug Saf. 2002;25(1):1-5. https:// doi.org/10.2165/00002018-200225010-00001.

10. Mason J, Pirmohamed M, Nunn T. Off-label and unlicensed medicine use and adverse drug reactions in children: a narrative review of the literature. Eur J Clin Pharmacol. 2012;68(1):21-8. https:// doi.org/10.1007/s00228-011-1097-1.

11. European Parliament. Regulation (EC) No 1901/2006 of the European Parliament and of the Council of 12 December 2006 on medicinal products for paediatric use and amending Regulation (EEC) No 1768/92, Directive 2001/20/EC, Directive 2001/83/EC and Regulation (EC) No 726/2004. Official Journal of the European Union; December 2006. p. 378/1-/19.

12. Hazell L, Shakir SA. Under-reporting of adverse drug reactions: a systematic review. Drug Saf. 2006;29(5):385-96. https://doi. org/10.2165/00002018-200629050-00003.

13. World Heatlh Organization. Safety of medicines, a guide to detecting and reporting adverse drug reactions, why health professionals need to take action. WHO/EDM/QSM/2002.2, Geneva. 2002. https://apps.who.int/iris/handle/10665/67378.

14. European Medicines Agency. Guideline on good pharmacovigilance practices (GVP) Annex I - Definitions (Rev 4) EMA/876333/2011 Rev 4*. Heads of Medicines Agencies; October 2017.

15. European Parliament. Directive 2010/84/EU of the European Parliament and of the Council of 15 December 2010 amending, as regards pharmacovigilance, Directive 2001/83/EC on the Community code relating to medicinal products for human use. Official Journal of the European Union; December 2010. p. 348/74 - /99.

16. The Uppsala Monitoring Centre. The use of the WHO-UMC system for standardized case causality assessment. World Health Organization - Uppsala Monitoring Centre. https://www.who. int/medicines/areas/quality_safety/safety_efficacy/WHOcausali ty_assessment.pdf.

17. U.S. DEPARTMENT OF HEALTH AND HUMAN SERVICES. Common Terminology Criteria for Adverse Events (CTCAE) version 5.0. November 27, 2017. p. 1-155.

18. European Medicines Agency. ICH Topic E 2 A Clinical safety data management: definitions and standards for expedited reporting. London: European Medicines Agency ICH Harmonised Tripartite Guideline; 1995.

19. European Medicines Agency. ICH Topic E 2 D Post approval safety data management. London: European Medicines Agency; 2004.

20. Smyth RM, Gargon E, Kirkham J, Cresswell L, Golder S, Smyth $\mathrm{R}$, et al. Adverse drug reactions in children - a systematic review. PLoS ONE. 2012;7(3):e24061. https://doi.org/10.1371/journ al.pone. 0024061 .

21. Turner S, Nunn AJ, Fielding K, Choonara I. Adverse drug reactions to unlicensed and off-label drugs on paediatric wards: a prospective study. Acta Paediatr (Oslo, Norway: 1992). 1999;88(9):965-8. 
22. Netherlands C-S. StatLine database. CBS - Statistics Netherlands. https://opendata.cbs.nl/statline/\#/CBS/nl/dataset/83384NED/table $? \mathrm{dl}=23629$. Accessed November 2019.

23. Takata GS, Mason W, Taketomo C, Logsdon T, Sharek PJ. Development, testing, and findings of a pediatric-focused trigger tool to identify medication-related harm in US children's hospitals. Pediatrics. 2008;121(4):e927-e935935. https://doi.org/10.1542/ peds.2007-1779.

24. Matlow AG, Cronin CM, Flintoft V, Nijssen-Jordan C, Fleming M, Brady-Fryer B, et al. Description of the development and validation of the Canadian paediatric trigger tool. BMJ Qual Saf. 2011;20(5):416-23. https://doi.org/10.1136/bmjqs.2010.041152.

25. European Medicines Agency. Guideline on good pharmacovigilance practices (GVP) Module VI-Collection, management and submission of reports of suspected adverse reactions to medicinal products (Rev 2). EMA/873138/2011 Rev 2: Heads of Medicines Agencies; 2017.

26. Vallano A, Cereza G, Pedros C, Agusti A, Danes I, Aguilera $\mathrm{C}$, et al. Obstacles and solutions for spontaneous reporting of adverse drug reactions in the hospital. Br J Clin Pharmacol. 2005;60(6):653-8. https://doi.org/10.111 1/j.1365-2125.2005.02504.x.

27. Coley KCPP, Janice L. PhD; Weber, Robert J. MS; Rice, Kristen BS, BA; Ramanujam, Rangaraj PhD, MBA; Sirio, Carl A. MD. Perceived Barriers in using a region-wide medication error reporting system. J Patient Saf. 2006;2(1):39-44.

28. Carleton BC, Smith MA, Gelin MN, Heathcote SC. Paediatric adverse drug reaction reporting: understanding and future directions. The Canadian journal of clinical pharmacology $=$ Journal canadien de pharmacologie clinique. 2007;14(1):e45-57.

29. Carleton B, Poole R, Smith M, Leeder J, Ghannadan R, Ross C, et al. Adverse drug reaction active surveillance: developing a national network in Canada's children's hospitals. Pharmacoepidemiol Drug Saf. 2009;18(8):713-21. https://doi.org/10.1002/pds.1772.

30. Wiktorowicz M, Lexchin J, Moscou K. Pharmacovigilance in Europe and North America: divergent approaches. Soc Sci Med (1982). 2012;75(1):165-70. https://doi.org/10.1016/j.socsc imed.2011.11.046.

31. Li X, Li H, Deng J, Zhu F, Liu Y, Chen W, et al. Active pharmacovigilance in China: recent development and future perspectives. Eur J Clin Pharmacol. 2018;74(7):863-71. https://doi. org/10.1007/s00228-018-2455-z.

32. Cliff-Eribo KO, Sammons H, Choonara I. Systematic review of paediatric studies of adverse drug reactions from pharmacovigilance databases. Exp Opin Drug Saf. 2016;15(10):1321-8. https ://doi.org/10.1080/14740338.2016.1221921.

33. Arencibia ZB, Sotomayor DN, Mollinedo NC, Choonara I, Manzano EF, Leyva AL. Adverse drug reactions in children in Camaguey Province, Cuba. Arch Dis Childh. 2010;95(6):474-7. https ://doi.org/10.1136/adc.2009.180786.

34. Barzaga Arencibia Z, Lopez Leyva A, Mejias Pena Y, Gonzalez Reyes AR, Fernandez Manzano E, Choonara I. Pharmacovigilance in children in Camaguey Province, Cuba. Eur J Clin Pharmacol. 2012;68(7):1079-84. https://doi.org/10.1007/s0022 8-012-1222-9.

\section{Affiliations}

\section{Anne T. M. Dittrich ${ }^{1,2}$. Jos M. T. Draaisma ${ }^{1}$ - Eugène P. van Puijenbroek ${ }^{3,4}$ - D. Maroeska W. M. te Loo ${ }^{2,5}$}

1 Department of Paediatrics, Radboud University Medical Centre, Radboud Institute for Health Sciences, Amalia Children's Hospital, Huispost 804, Postbus 9101, 6500 HB Nijmegen, the Netherlands

2 Department of Pharmacology and Toxicology, Radboud University, Nijmegen, the Netherlands

3 Netherlands Pharmacovigilance Centre Lareb, 's-Hertogenbosch, the Netherlands
4 Unit of Pharmacotherapy, Epidemiology and Economics, Groningen Research Institute of Pharmacy, University of Groningen, Groningen, the Netherlands

5 Department of Paediatric Haematology, Radboud University Medical Centre, Radboud Institute for Health Sciences, Amalia Children's Hospital, Nijmegen, the Netherlands 